\title{
Development of a Material Mixing Method Based on Evolutionary Structural Optimization*
}

\author{
Seog-Young HAN** and Soo-Kyoung LEE***
}

\begin{abstract}
This paper suggests a material mixing method to mix several materials in a structure. This method is based on ESO (Evolutionary Structural Optimization), which has been used to optimize topology of only one material structure. In this study, two criterions for material transformation and element removal are implemented for mixing several materials in a structure. Optimal topology for a multiple material structure can be obtained through repetitive application of the two criterions at each iteration. Two practical design examples of a short cantilever are presented to illustrate validity of the suggested material mixing method. It is found that the suggested method works very well and a multiple material structure has more stiffness than one material structure has under the same mass.
\end{abstract}

Key Words: Material Mixing Method, Multiple Material, Topology Optimization, Transformation Line, Removal Line, Evolutionary Structural Optimization

\section{Introduction}

Topology optimization is very useful in making a conceptual design when considering weight and cost in the early design stage. An important development in topology optimization was made by Bendsøe and Kikuchi ${ }^{(1)}$ who proposed the homogenization method, in which a material with an infinite number of microscale voids is introduced, and the optimization problem is defined by seeking the optimal porosity of a porous medium using an optimality criterion. Mlejnek et al. ${ }^{(2)}$ has accomplished shape and topology optimization using a simple energy method and a special type of function, that is, Kreisselmeier-Steinhauser function $^{(3)}$ for calculating effective properties.

Recently, a simple method for shape and topology optimization, called Evolutionary Structural Optimization (ESO), has been proposed by Xie and Steven ${ }^{(4)}$ and $\mathrm{Chu}^{(5)}$, which is based on the concept of gradually removing redundant elements of the low stressed part of the material from a structure to achieve an optimal design.

In the area of MEMS, topology optimization techniques have also been actively applied. Especially, one

${ }^{*}$ Received 8th March, 2004 (No. 04-5031)

** School of Mechanical Engineering, Hanyang University, 17 Haengdang-Dong, Sungdong-Gu, Seoul 133-791, Korea. E-mail: syhan@email.hanyang.ac.kr

*** Mechanical Engineering Center M202-1, Hanyang University, 17 Haengdang-Dong, Sungdong-Gu, Seoul 133 791, Korea. E-mail: 11s1k1@ hanmail.net of the thermal actuators, microgripper, has been designed by a bimorph structure ${ }^{(6)}$ consisted of two materials with the different thermal strains, so that the improvement of its performance has been achieved by controlling the direction and magnitude of overall displacement effectively.

In this study, a material mixing method was suggested in order to obtain an optimal topology of a multiple material structure with the maximum stiffness or displacement constraints under a static load based on ESO.

\section{Material Mixing Method}

The explanation of the material mixing method for a bimorph structure is given here in brief. Let the larger and the smaller stiffness and density of the two materials set material 1 and material 2, respectively. First of all, consider a design region made of material 1. Apply both the transformation and the removal lines for material transformation and element removal, respectively. As the first step, the elements having lower level of strain energy than the transformation line are transformed into material 2 . In the second step, the elements having a lower level of strain energy than the removal line, which is established as the lower level of strain energy than the transformation line, are removed. An optimal topology can be obtained by repetition of this procedure at each iteration.

\subsection{Transformation and removal line}

2.1.1 Transformation line The transformation line was defined as Eq. (1) provided for a journal paper ${ }^{(7)}$ in this study. And the efficiency factor $\alpha$ is defined as 
Eq. (2), which is the ratio of the strain energy in each element, $u^{e}$, to the maximum strain energy, $u^{\max }$, in entire structure. If $\alpha$ of a certain element is smaller than the transformation line, that is, Eq. (3) is satisfied, the element is transformed from material 1 to material 2,

$$
\begin{aligned}
& T L=\alpha^{\min }+\Delta \alpha^{T L} \times\left(\frac{\text { vol }_{\text {present }}}{\text { vol }_{\text {initial }}}\right)^{\eta} \\
& \alpha^{e}=\frac{u^{e}}{u^{\max }} \\
& \alpha^{e} \leq T L
\end{aligned}
$$

where, the threshold ratio $\Delta \alpha$ can be selected as very small value depending on the problem since it controls the range of the elements to be transformed. The elements on the area of diagonal lines under the transformation line as shown in Fig. 1 are to be transformed. This concept is similarly applied to the removal line. Penalty factor $\eta$ is induced to obtain an optimal topology effectively by controlling the range of the transformed elements, that is, gradually reducing the range as the number of iteration increases.

2.1.2 Removal line The removal line was defined as Eq. (4) in this study. If $\alpha$ of a certain element is smaller than the removal line, that is, Eq. (5) is satisfied, the element is removed from the structure. The threshold ratio and the penalty factor were similarly applied for determination of the removal line. The removal line under the transformation line was obtained by controlling the sizes of the threshold ratio and the penalty factor. It can be seen in Fig. 2 that the number of the removed elements

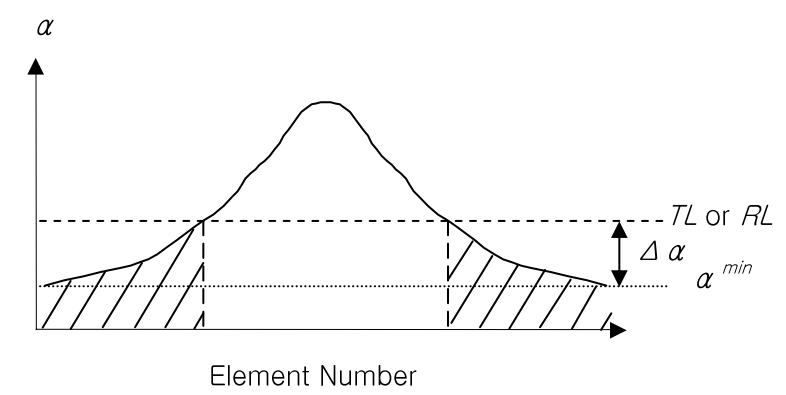

Fig. 1 Transformation and removal line

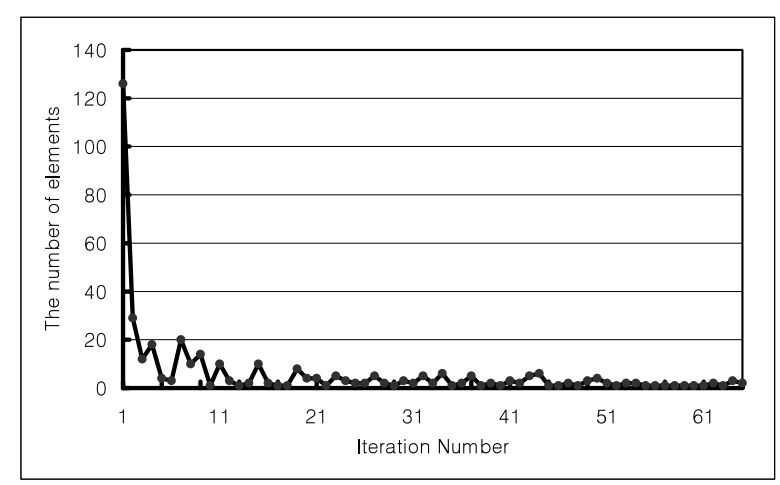

Fig. 2 Reduction of the number of the removed elements for the example in section 3.1 is reducing as iteration goes on. Inducing the threshold ratio and penalty factor to the transformation and removal lines, we varied the removal ratio from $20 \%$ at the beginning to $1 \%$ at the last iteration.

$$
\begin{aligned}
& R L=\alpha^{\mathrm{min}}+\Delta \alpha^{R L} \times\left(\frac{\text { vol }_{\text {present }}}{\text { vol }_{\text {initial }}}\right)^{\eta} \\
& \alpha^{e} \leq R L
\end{aligned}
$$

\subsection{Procedure of topology optimization}

Optimal topology based on ESO has been obtained through stress analysis by the finite element method, material transformation by the transformation line and element removal by the removal line. The flowchart of topology optimization process is shown in Fig. 3. This procedure is iterated until the prescribed mass or deflection constraint is satisfied.

\section{Examples}

\subsection{A short cantilever beam subject to a concen- trated force at the center of free end}

When a short cantilever shown in Fig. 4 was subjected to a concentrated force of $300 \mathrm{kN}$ at the center of free end, optimal topologies of single and two-multiple material structures were obtained using the suggested material mixing method. The quadrilateral element was used and the beam was divided into a grid of $32 \times 20$. The used elastic modulii are listed in Table 1. It was assumed that the elastic modulus of material 2 is $40 \%$ of material 1 .

In order to investigate the topology optimization process as the materials are mixing, topology optimizations were performed for the following four cases. Those are; (1) a single material structure of material 1 with $50 \%$ mass

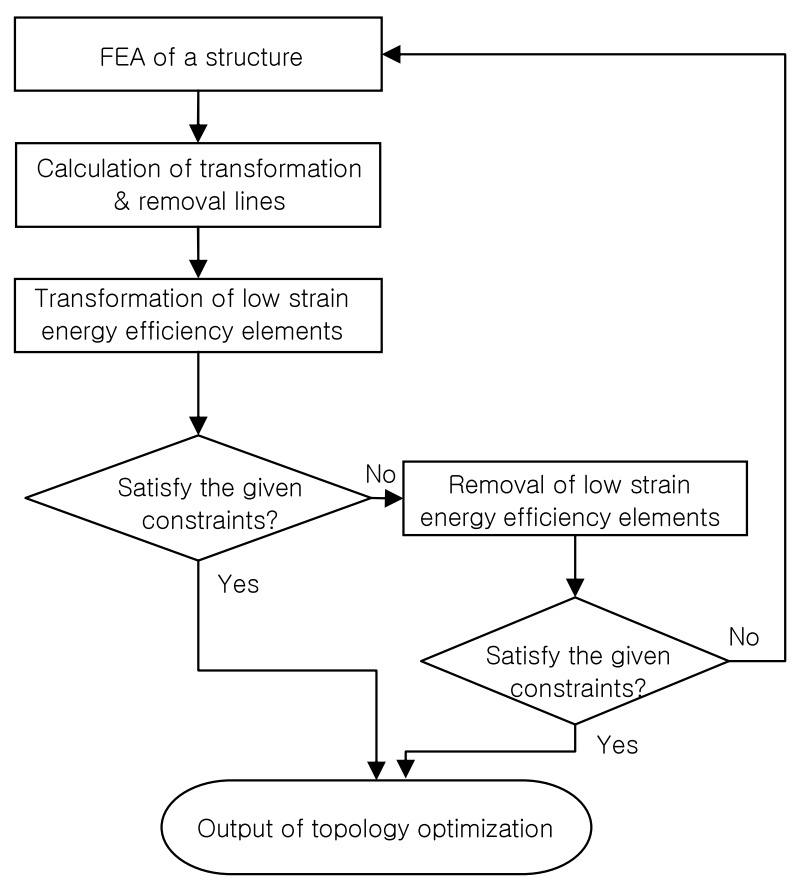

Fig. 3 Flowchart of topology optimization process 


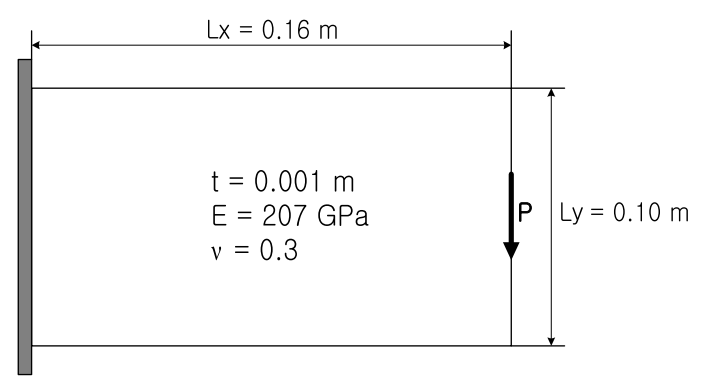

Fig. 4 Conditions of a short cantilever

Table 1 Material properties

\begin{tabular}{c|c|c|c}
\hline Material & Young's Modulus & Density & Poison's Ratio \\
\hline Material 1 & $207 \mathrm{GPa}$ & $7280 \mathrm{~kg} / \mathrm{m}^{3}$ & 0.3 \\
\hline Material 2 & $82.8 \mathrm{GPa}$ & $3128 \mathrm{~kg} / \mathrm{m}^{3}$ & 0.3 \\
\hline
\end{tabular}

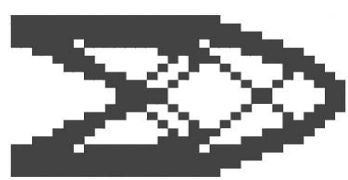

(a)

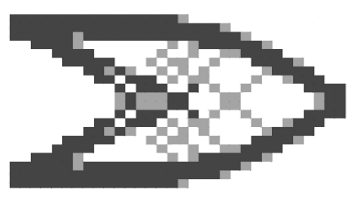

Material $1 \quad$ Material 2

(b)

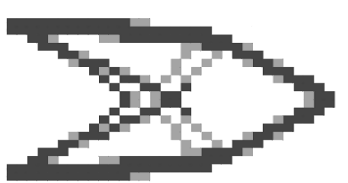

(c)

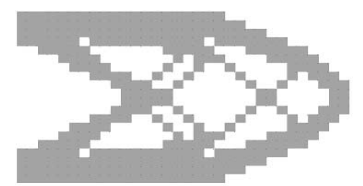

(d)
Fig. 5 Optimized topology obtained for; (a) case (1), (b) case (2), (c) case (3), (d) case (4)

constraint of initial mass of material 1, (2) two-multiple material structure with $40 \%$ mass constraint, (3) twomultiple material structure with $30 \%$ mass constraint, and (4) a single material structure of material 2 with $20 \%$ mass constraint.

The obtained optimal topology of each case is shown in Fig. 5. The optimal topologies of the cases (1) and (4) are the same as those obtained using $\mathrm{ESO}^{(8)}$. Figure 5 (b) and (c) indicate the material mixing process of two materials properly as the mass of a structure decreases. And the deflections tend to increase as the mass of a structure decreases. Therefore, it can be verified that topology optimization was properly performed.

\subsection{A short cantilever beam subject to a concen- trated force at the right end of free end}

When a short cantilever beam is subjected to a concentrated force of $300 \mathrm{kN}$, and the maximum deflection is limited to $0.8 \mathrm{~mm}$ at the right end of free end instead of the center of free end in Fig. 4, optimal topologies of single, two and three multiple structures were obtained using the suggested material mixing method. The elastic modulii used in this example are listed in Table 2. Since this
Table 2 Material properties

\begin{tabular}{c|c|c|c}
\hline Material & Young' s Modulus & Density & Poison's Ratio \\
\hline Material 1 & $207 \mathrm{GPa}$ & $7280 \mathrm{~kg} / \mathrm{m}^{3}$ & 0.3 \\
\hline Material 2 & $144.9 \mathrm{GPa}$ & $5474 \mathrm{~kg} / \mathrm{m}^{3}$ & 0.3 \\
\hline Material 3 & $82.8 \mathrm{GPa}$ & $3128 \mathrm{~kg} / \mathrm{m}^{3}$ & 0.3 \\
\hline
\end{tabular}

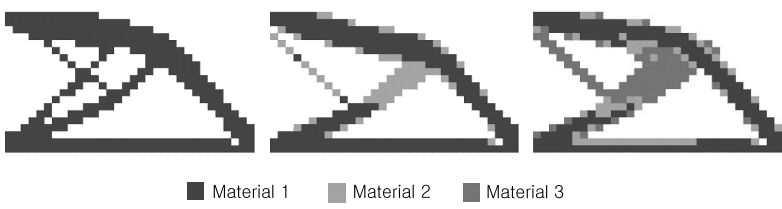

(a)

(b)

(c)

Fig. 6 Optimized topology obtained for a structure consisted of; (a) one material (b) two materials (c) three materials

Table 3 Final masses of optimal topologies

\begin{tabular}{c|c|c|c}
\hline Constitution & 1 Material & 2 Materials & 3 Materials \\
\hline Mass & $0.0500 \mathrm{~kg}$ & $0.0475 \mathrm{~kg}$ & $0.0449 \mathrm{~kg}$ \\
\hline
\end{tabular}

example dealt with a three multiple material structure, two transformation and one removal line were implied.

The obtained three optimal topologies are shown in Fig. 6. And the final masses were obtained under the maximum deflection constraint as shown in Table 3. Since the optimal topology of a three-multiple material structure has the smallest mass, it is known that a three-multiple material structure has the largest stiffness compared with the other optimal structures under the same mass constraint.

\section{Conclusions}

In this study, a material mixing method has been developed based on ESO in order to obtain an optimal topology of a multiple material structure. From the results of the presented examples, the following conclusions are obtained:

(1) Introducing the concepts of the transformation and removal lines, we obtained optimal topologies by iterating material transformation and removal.

(2) Inducing the threshold ratio and penalty factor to the transformation and removal lines, we varied the removal ratio from $20 \%$ at the beginning to $1 \%$ at the last iteration. Therefore, it is known that the convergence rate in this study is much faster than the ordinary ESO, of which removal ratio is usually chosen as 1 or $2 \%$ in static problems.

( 3 ) It was found that the optimal topology of multiple materials has larger stiffness than that of single material under the same mass.

\section{References}

( 1 ) Bendsøe, M.P. and Kikuchi, N., Generating Optimal Topologies in Structural Design Using a Homogenization Method, Comp. Meth. Appl. Mech. Engng., Vol.71 (1988), pp.197-224. 
( 2 ) Mlejnek, H.P. and Schirmacher, R., An Engineer's Approach to Optimal Material Distribution \& Shape Finding, Comp. Meth. Appl. Mech. Engng., Vol.106 (1993), pp.1-26.

( 3 ) Kreisselmeier, G. and Steinhauser, R., Systematic Control Design by Optimizing a Vector Performance Index, IFAC Symp. Computer Aided Design of Control Systems, Zürich, Switzerland, (1979).

(4) Xie, Y.M. and Steven, G.P., A Simple Evolutionary Procedure for Structural Optimization, Comput. Struc., Vol.49 (1993), pp.885-896.

( 5 ) Chu, D.N., Xie, Y.M., Hira, A. and Steven, G.P., Evolutionary Structural Optimization for Problems with Stiffness Constraints, Finite Elements in Analysis and
Design, No.21 (1996), pp.239-251.

( 6 ) Yin, L. and Ananthasuresh, G.K., A Novel Topology Design Scheme for the Multi-Physics Problems of Electro-Thermally Actuated Compliant Micromechanisms, Sensors and Actuators A: Physical, Vol.97-98 (2002), pp.599-609.

( 7 ) Qing, L., Steven, G.P., Querin, O.M. and Xie, Y.M., Structural Topology Design with Multiple Thermal Criteria, Engineering Computations, Vol.17 (2000), pp.715-734.

( 8 ) Han, S.Y., An Improved Element Removal Method for Evolutionary Structural Optimization, KSME International Journal, Vol.14, No.9 (2000), pp.913-919. 